papers is a monograph by Hertig and Rock on early human embryos. In recent years these two authors have added almost as much to our knowledge of the earliest stages of man's development as have all previous workers put together. Their present study deals with two human ova of the pre-villous stage, and whose ages are estimated to be about eight and nine days respectively. Like their previous observations, this new study is bound to become text-book material. Finally, the volume contains a review of the histogenesis of cartilage and bone, by the late Dr. George L. Streeter, formerly head of the Depart. ment of Embryology, Carnegie Institution of Wash. ington, Baltimore, and to whose inspiration we largely owe the high standard of the journal in which this posthumous paper appears.

\section{Electricity as an Aid to Productivity}

A conference on "Electricity as an Aid to Productivity", organised by the Institution of Electrical Engineers, will be held in the Lecture Theatre of the Institution during November 15-16. The conference is intended mainly for executives in both large and small factories who are responsible for production, including managers, production engineers and factory engineers, and will consist of a series of lectures on the following five topics: motive power in the factory; industrial heating processes; welding applications; the handling and inspection of materials; lighting, heating, ventilation, etc. The lectures have been planned from the point of view of the user, and will deal, in general terms, with what electricity can do to increase production rather than with technical details. A report of the proceedings of the conference will be published later (price 6s.). . Tickets for the conference can be obtained from the Secretary of the Institution of Electrical Engineers, Savoy Place, London, W.C.2.

\section{Australian Journal of Applied Science}

The first issue of a new quarterly journal, the Australian Journal of Applied Science, published by the Commonwealth Scientific and Industrial Research Organisation, appeared in March 1950 (1, No. 1; pp. 132; Melbourne: Government Printer; $30 s$. a year). It is intended as a medium for the publication of the results of original investigations in the broad field of applied science and is complementary to the Australian Journal of Scientific Research (A, Physical ; B, Biological), of Agriculture Research, and of Marine and Freshwater Research, all three of which are published by the same Organisation. The first number covers a wide field with ramifications into electrical technology, applied physics and chemistry, and the technology of timber. There is a detailed study of the setting of synthetic resin and casein glues by means of the viscometer ; very full data concerning the mechanical properties of red tulip oak, a tree abundant in Queensland; and an assessment of the value of various tannins, that can be extracted from Australian trees, for the production of adhesives by condensation with formaldehyde. There is also an interesting account of the production of metal surfaces suitable for use as standards of roughness or surface finish; strips of chromium controlled in width, spacing and hoight are electrolytically deposited on the lapped surface of a steel block. Improvements in the production of graticules by the use of metal films evaporated on to the glass surface are also described, and there is a series of five papers on the special treatments that must be applied to electrical equipment to prevent its rapid deterioration under tropical conditions. These papers record a long series of investigations in which many organisations collaborated during the years $1943-46$, and the most vital parts of the information are therefore already known to the radio industry. It is, however, very useful to have a systematic and detailed survey easily accessible for reference. Those who are interested in the physics and chemistry of water-repellent materials for the treatment of glass and ceramic insulators will find much new information in studies of the efficacy of quatemary ammonium as well as organo-silicon compounds used for this purpose. The new Journal will be welcomed as an important contribution to the literature of applied science.

\section{Progress in Theoretical Physics}

ONE of the most remarkable things that came to light after the end of hostilities in 1945 was the emergence during the War of a vital and important school of theoretical physics in Japan. It is so active that it has been able to found a journal devoted almost exclusively to theoretical physics, which must take its place on the shelves of any complete scientific library, side by side with other important learned journals. Progress in The retical Physics (published by Akitaya Co., Ltd., Osaka, Kyoto, Japan) is edited by $H$. Yukawa and printed in English. It started publication in June 1946; but unfortunately it has not, up to the present, been easy to obtain copies in Great Britain. The difficulties seem to be connected with export restrictions imposed by the occupying Powers. It is to be hoped that so important a journal will soon be readily available, and that an adequate stock of back numbers is in existence. The journal is mostly devoted to papers on the quentum theory of fields - as is natural in a country where physics is strongly influenced by the work of Yukawa and Tomonaga - but there are also papers on ferromagnetism and astrophysics in the early issues. It contains many important papers ; Tomonaga's classic paper on the covariant formulation of quantum field theories, for example, appeared in the second issue (August-September 1946), to be followed by developments of the idea in later papers. The journal advertises itself on the inside cover as mainly concerned with papers on theoretical physics, which are related to quantum mechanics, statistical mechanics, the theory of relativity and the theory of elementary particles-showing that its editorial board is keenly aware of the direction in which vital advances are being made in physics. Experimental papers intimately connected with contemporary theoretical physics are also eligible for inclusion.

\section{Educational Times}

The Educational Times, which was first published in 1847, is now to become a digest of current educa. tional literature and will be published three times a year, in February, June and October (from the College of Preceptors, 2 and 3 Bloomsbury Square, London, W.C.1. 1s.). This change has been brought about because it is felt that, with the present-day spate of periodicals dealing wholly or partly with educational topics, the difficulty of the teacher and interested layman is not that he has too little to read, but rather that he has too much and that it is so widely dispersed. It is hoped, therefore, that the Educational Times in a new form, and with a new purpose, may satisfy the need of those who wish 